\title{
Inflammatory stress promotes lipid accumulation in the aorta and liver of SR-A/CD36 double knock-out mice
}

\author{
HONG LIU ${ }^{1}$, QING LIU ${ }^{1}$, HAN LEI $^{2}$, XIULAN LI ${ }^{1}$ and XIAN CHEN ${ }^{1}$ \\ ${ }^{1}$ Clinical Research Center, and ${ }^{2}$ Department of Cardiology, The First Affiliated Hospital, \\ Chongqing Medical University, Chongqing 400016, P.R. China
}

Received June 4, 2010; Accepted August 19, 2010

DOI: $10.3892 / \mathrm{mmr} .2010 .351$

\begin{abstract}
Macrophage scavenger receptors (SRs), particularly type A SR (SR-A) and CD36, have been implicated in binding and internalizing modified forms of low-density lipoprotein (LDL) through a mechanism not regulated by cellular cholesterol content, resulting in cholesterol accumulation and foam cell formation. To test the impact of the absence of these receptors on lipid-mediated injuries in aortas or livers under inflammatory stress, C57BL/6 mice with knocked out SR-A and CD36 (SR DKO) were administered a combination of a high-fat diet and casein injection for 14 weeks. We analyzed the lipid accumulation in the aortas and livers, assessing lipid droplets, circulating inflammatory cytokines, serum lipid levels and gene expression of cholesterol uptake. Lipid accumulation in the aortas and livers of the inflammatory mice were assessed by morphologic and quantitative assay of cholesterol ester in the livers. The SR DKO mice under inflammatory stress showed an increased level of serum amyloid A, but did not exhibit any significantly increased levels of TNF- $\alpha$ and IL-6, while morphological analysis revealed a significant increase in lipid accumulation in the aortas and livers of the inflammatory mice. Although SR DKO mice exhibit substantially reduced inflammatory cytokine factors, loss of the SR pathway does accelerate lipid accumulation through LDL receptor pathways to lipid accumulation. These data suggest that targeted inhibition of SR pathways in vivo may promote lipid-mediated injuries in aortas and livers under inflammatory stress.
\end{abstract}

\section{Introduction}

Macrophage scavenger receptors (SRs) contribute both to early foam cell formation and to the progression of atherosclerosis

Correspondence to: Dr Qing Liu, Clinical Research Center, The First Affiliated Hospital, Chongqing Medical University, No. 1 Yixueyuan Road, Chongqing 400016, P.R. China

E-mail: qingliu5566@yahoo.cn

Keywords: sterol regulatory element-binding protein 2, inflammatory stress, scavenger receptor, lipid accumulation, low-density lipoprotein receptor
(AS). Knock-out of either of the two SR family members, type A SR (SR-A) or CD36, has been previously shown to reduce oxidized low-density lipoprotein (LDL) uptake in vitro and atherosclerotic lesions in vivo (1). Kunjathoor et al (2) showed that SR-A and CD36 account for 75-90\% of the degradation of LDL modified by acetylation or oxidation of the majority of lipid accumulation in macrophages exposed to oxidized LDL. Although alternative models of AS with $\mathrm{ApoE}^{-/}$, not involving SRs, have been extensively investigated in vitro, previous studies have shown that deficiency of SR-A or CD36 did not prevent macrophage foam cell formation in vivo (3-5). However, other studies have revealed that the absence of SR-A was protective ( $32 \%$ decrease in the lesion). The combined absence of CD36 and SR-A provided no further protection in individuals of either gender (6).

Lipid disturbances play a major role in foam cell formation. A recent study in our laboratory indicated that inflammatory stress may increase gene expression involving lipid metabolism in vitro (7). An alternative role for the absence of scavenger receptors in promoting atherogenesis has been elucidated in the background of $\mathrm{ApoE}^{-/-}$mice in many studies, but the contribution of deleting scavenger receptors under inflammatory stress to lipid accumulation is uncertain. Thus, we further hypothesized that the absence of SRs enhances the dysregulation of lipid metabolism in an inflammatory animal model. In the present study, SR DKO mice treated with a casein injection were used to investigate lipid accumulation in aortas and livers.

The liver plays a key role in the uptake of cholesterol via the LDL receptor (LDLr) pathway, which is tightly regulated by a feedback system that maintains intracellular cholesterol homeostasis (8). Regulation of cholesterol biosynthesis occurs via a family of transcription factors designated as sterol regulatory element-binding proteins (SREBPS). There are three known members of the SREBP family: SREBP1a, SREBP1c and SREBP2; the last one is regarded as the main regulator of cholesterologenesis. Thus, SREBP2 may represent a suitable candidate for the regulation of cholesterol synthesis in diseases associated with lipid accumulation.

LDLr gene expression is predominantly regulated by a negative-feedback mechanism that depends on the mediation of intracellular cholesterol concentration by SREBPs and SREBP cleavage activating protein (SCAP) (9). SREBP genes encode membrane proteins that are bound to endoplasmic 
reticulum (ER) membranes and serve as precursors for active transcription factors, called mature or nuclear SREBPs. Transcription factor release is controlled by SCAP, which escorts the SREBP precursor from ER to the Golgi apparatus. Regulation of cholesterol biosynthesis occurs via a family of transcription factors designated as SREBPs. SREBP2 is a transcription factor regarded as the main regulator of cholesterol homeostasis. When the cellular cholesterol level is low, the C-terminal regulatory domain of the SREBP precursor interacts with SCAP and, in consequence, the molecule is transported to Golgi apparatus where the SREBP precursor is clipped by site-1-protease. Then, site-2-protease clips the $\mathrm{N}$-terminal SREBP intermediate releasing the mature transcription factor that activates LDLr gene transcription. Upon translocation to the nucleus, it binds to SRE sequences within the promoters of target genes and, in consequence, enhances their transcription. The opposite situation occurs in the case of sterol overload. SREBP remains bound to endoplasmic reticulum membranes, where they are inactive as transcription factors. Expression of target genes ceases and the cellular cholesterol level decreases. Intracellular cholesterol is limited by the down-regulation of LDLr (10).

Recently, we showed that inflammatory stress exacerbated lipid dysregulation in in vitro experiments and found increased lipid-related gene expression in mammalian cells with increased lipid accumulation (7). The present study was designed to provide an explanation for the accumulation of cholesterol in aortas and livers by studying the effects of inflammatory stress on LDLr-mediated cholesterol uptake in aortas and livers of SR DKO mice administered a combination of a high-fat diet and subcutaneous injection of $10 \%$ casein.

\section{Materials and methods}

Animal model. Male SR DKO mice of C57BL/6 genetic background were bred under protocols in accordance with the Chongqing Medical University's Subcommittee on Research Animal Care and Use of Laboratory Animals. All mice were kept in a pathogen-free environment in autoclaved filter-top cages with autoclaved water and on a 12-h light/dark cycle. At 6-8 weeks of age, the mice fed a high-fat diet containing $21 \%$ (w/w) buttermilk, $1.25 \%(\mathrm{w} / \mathrm{w})$ cholesterol, $19.5 \%(\mathrm{w} / \mathrm{w})$ casein and $0.5 \%(\mathrm{w} / \mathrm{w})$ sodium cholate were randomly assigned ( $n=6 /$ group) by subcutaneous injections of either casein or NS for up to 14 weeks. At termination, the aorta, liver and serum were collected and frozen at $-80^{\circ} \mathrm{C}$. Serum amyloid A (SAA) (Rapid Biological Ltd.), TNF- $\alpha$ (R\&D Ltd., USA) and IL-6 (R\&D Ltd.) levels were determined by enzyme-linked immunosorbent assay. Total cholesterol and LDL (Rongsheng Ltd., China) were measured by enzymatic assay according to the manufacturer's protocol in the kit. The results are expressed as the mean \pm standard deviation (SD). Aorta and liver samples were used for histological assessments.

Observation of lipid accumulation. The lipid accumulation in aortas and livers was evaluated by Oil Red $\mathrm{O}$ staining. Briefly, samples were fixed with $5 \%$ formalin solution and then stained with Oil Red O for $15 \mathrm{~min}$. Finally, the samples were counterstained with hematoxylin for $2 \mathrm{~min}$. Results were examined by light microscopy.
Table I. Mouse TaqMan primers for real-time PCR.

Genes Mouse TaqMan primers

SCAP

5'-TGGAGCTTTTGAGACTCAGGA-3' sense 5'-TCGATTAAGCAGGTGAGGTCG-3' antisense

SREBP-2

5'-TGAAGGACTTAGTCATGGGGAC-3' sense

5'-CGCAGCTTGTGATTGACCT-3' antisense

LDLr

5'-GAAGTCGACACTGTACTGACCACC-3' sense

5'-TGACTCAGACGAACAAGGCTG-3' antisense

18SrRNA

5'-CCTGGCACCCAGCACAAT-3' sense
5'-GCCGATCCACACACGGAGTACT-3' antisense

Quantitative measurement of cholesterol ester in the liver. Quantitative measurement of total and free cholesterol in the liver was analyzed using the method described by Gamble et al (11). In brief, samples were collected and lipids were extracted by the addition of $1 \mathrm{ml}$ chloroform/methanol (2:1). The lipid phase was collected, dried in a vacuum and then dissolved in 2-propanol containing 10\% Triton X-100. The concentrations of total and free cholesterol were analyzed using a standard curve and normalized by total protein from the liver tissues. The concentration of cholesterol ester was calculated using total cholesterol minus free cholesterol.

Isolation of total RNA and real-time reverse transcriptase polymerase chain reaction for gene expression. Total cellular RNA was extracted from frozen aortas or liver homogenates using the guanidinium-isothiocyanate-phenol/chloroform method, and the procedure was performed as described in the manufacturer's protocol (Takara). RNA concentration was determined from the absorbance at $260 \mathrm{~nm}$, and all samples had 260/280 $\mathrm{nm}$ absorbance ratios of 2.0 . The cDNA was used as a template in the RT-PCR reaction with housekeeping 18SrRNA gene as an internal control. The reverse transcriptional reaction was set up using a kit from ABI. Aorta and liver RNA $(1 \mu \mathrm{g})$ from each mouse was reverse-transcribed, and quantitative RT-PCR analysis of three genes related to cholesterol uptake was performed in an IQ5 using SYBR Green dye according to the manufacturer's protocol. All of the primers (Sangon, China) were designed by Primer Express Software V2.0, as shown in Table I. PCR conditions were 50 cycles of $95^{\circ} \mathrm{C}$ for $10 \mathrm{~min}, 95^{\circ} \mathrm{C}$ for $20 \mathrm{sec}$ and $55^{\circ} \mathrm{C}$ for $20 \mathrm{sec}$, then $72^{\circ} \mathrm{C}$ for $5 \mathrm{~min}$. Results for gene expression are expressed as mean fold value \pm SD over 18 SrRNA. Data analysis was performed using $\Delta \Delta \mathrm{Ct}$-based fold-change calculations.

Immunohistochemical staining. Frozen sections $(5-\mu \mathrm{m})$ were cut from the aortas or livers of SR DKO mice, air-dried and fixed in ethanol. Slides were washed with Tris-buffered saline and $0.3 \%$ Tween (TBST, pH 7.4). Endogenous peroxidase activity was blocked with $0.5 \%$ hydrogen peroxide in methanol for $10 \mathrm{~min}$. Sections were washed 3 times with TBST and 
A
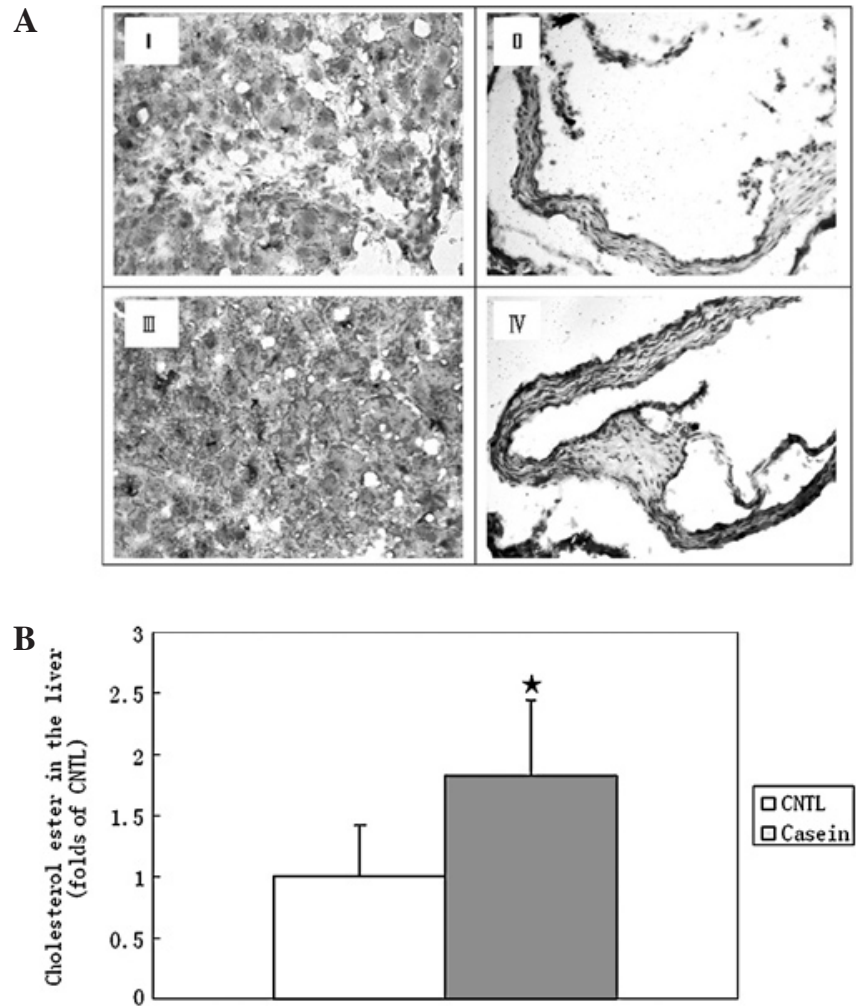

Figure 1. Lipid accumulation in the livers of SR DKO mice treated or not with a injection. SR DKO mice were administered a high-fat diet for 14 weeks without (CNTL) or with a 10\% casein injection (Casein). (A) The lipid accumulation in the livers (I and III, original magnification x400) and aortas (II and IV, original magnification x200) were assessed by Oil Red O staining using $5-\mu \mathrm{m}$ sections. The concentrations of cholesterol ester (B) in the livers of SR DKO mice, either receiving a casein injection or not, were measured as described in Materials and methods. Results represent the mean \pm SD $(\mathrm{n}=6) .{ }^{*} \mathrm{P}<0.01$ vs. control.

blocked with $10 \%$ serum in TBST for 10 min. Primary antimouse polyantibodies of SREBP2, SCAP and LDLr (Abcam, UK) were added at a 1:250 dilution in TBST. Sections were incubated at room temperature for $1 \mathrm{~h}$ in a humidified chamber and washed 3 times with TBST. Horseradish peroxidase antirabbit antibody for SCAP, LDLr (1:300 dilution) or horseradish peroxidase anti-mouse antibody for SREBP2 (1:300 dilution) (both from Santa Cruz Biotechnologies, Santa Cruz, CA, USA) were used as the secondary antibody, and sections were incubated for $30 \mathrm{~min}$ before they were washed 3 times with TBST. Horseradish peroxidase activity was detected with use of a DAB solution (Zhongshan, China). The reaction was stopped with tap water. Results were examined by light microscopy. Targeted protein stained brown.

Statistical analysis of the data. In all of the experiments, results are presented as the mean $\pm \mathrm{SD}$. Statistical significance of differences between groups was assessed by one-way analysis of variance, followed by the $t$-test. Differences between groups were considered significant at a P-value $<0.01$.

\section{Results}

SR DKO mice administered a combination of a high-fat diet and subcutaneous injection of $10 \%$ casein for 14 weeks
A

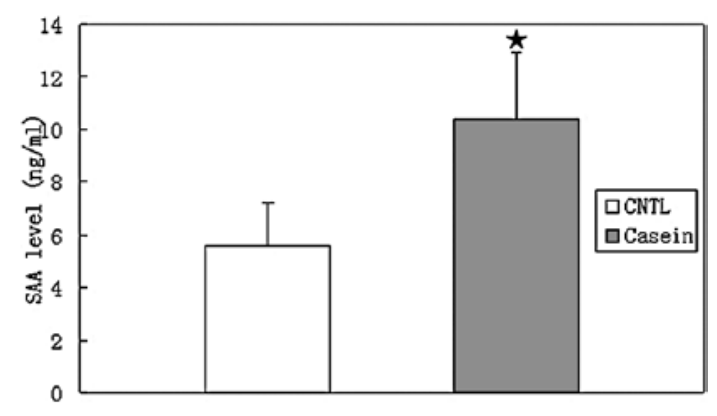

B

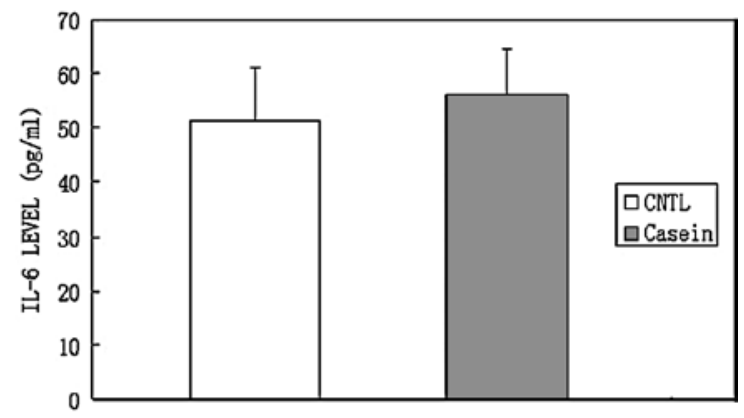

C

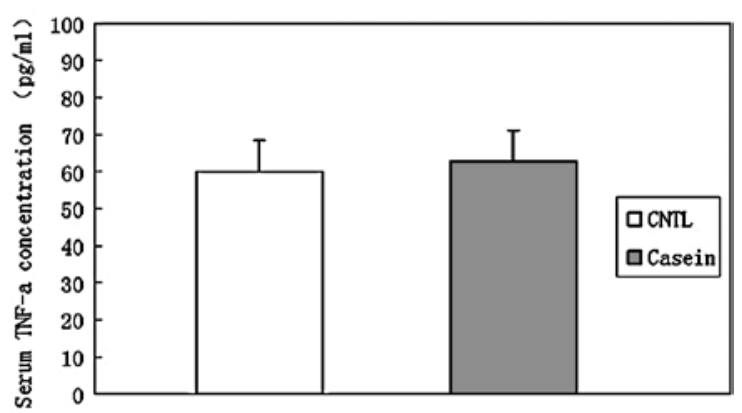

Figure 2. The production of inflammatory cytokines in the serum of SR DKO mice, in the absence or presence of casein injection. SR DKO mice were fed a high-fat diet for 14 weeks, without (CNTL) or with a $10 \%$ casein injection (Casein). The level of SAA (A), IL-6 (B) and TNF- $\alpha$ (C) in the serum of SR DKO mice was assessed by enzyme-linked immunosorbent assay. Results represent the mean $\pm \mathrm{SD}(\mathrm{n}=6) .{ }^{*} \mathrm{P}<0.01$ vs. control.

displayed an increase in lipid droplets in the livers (Fig. 1A, I and III) and aortas (Fig. 1A, II and IV). Furthermore, the cholesterol ester concentration in the livers (Fig. 1B) of the inflammatory mice was also higher than that in the control mice.

The inflammatory mice displayed a $>2$-fold increased level of serum SAA (Fig. 2A) compared to the controls, suggesting that inflammatory stress was successfully induced. However, we also measured levels of additional serum inflammatory cytokines. Serum IL-6 (Fig. 2B) and TNF- $\alpha$ (Fig. 2C) concentrations in the inflammatory mice were not significantly increased compared to the control animals. Serum total cholesterol (Fig. 3A) and LDL (Fig. 3B) concentrations in the serum of inflammatory mice were significantly lower than the levels found in the controls.

Taking into consideration that hypocholesterolemia and increased lipid accumulation in the aortas and livers may also be due to the up-regulation of SREBP2 and its target gene (LDLr) and its regulatory gene (SCAP) under inflammatory stress, our results indicated that mRNA expression (Fig. 4A) 


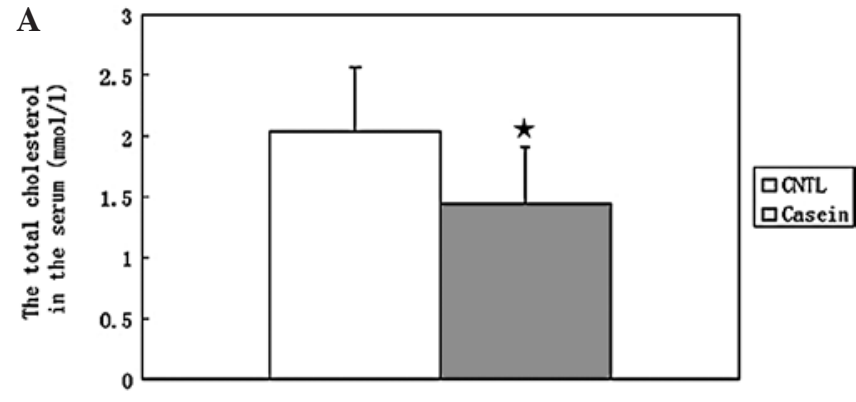

B

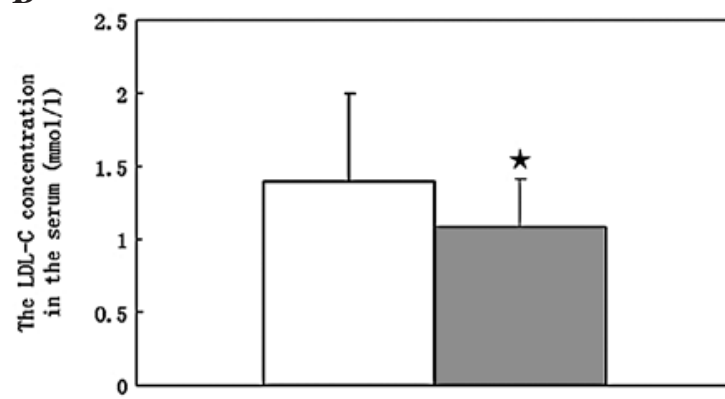

Figure 3. Lipid concentrations in the serum of SR DKO mice either receiving or not a casein injection. SR DKO mice were fed a high-fat diet for 14 weeks, without (CNTL) or with a 10\% casein injection (Casein). The concentrations of total cholesterol (A) and LDL-C (B) in the serum of SR DKO mice were measured by enzymatic assay. Results represent the mean $\pm \mathrm{SD}(\mathrm{n}=6)$. ${ }^{*} \mathrm{P}<0.01$ vs. control.

and mature protein in both livers (Fig. 4B) and aortas (Fig. 4C) of the inflammatory animals were significantly higher than that found in the control animals.

\section{Discussion}

SRs provide a high affinity uptake mechanism for the accumulation of lipid derived from modified LDL; it is widely believed that they play a critical role in lipid accumulation. Kunjathoor et al demonstrated that SR-A and CD36 are critical contributors to modified lipoprotein uptake in macrophages in vitro. In the absence of both receptors, lipoprotein uptake and degradation were reduced by $75-90 \%$ (2). We, therefore, sought to explore whether there may be other receptor pathways that mediate unmodified LDL uptake and their contribution to cholesteryl ester accumulation and lipid-mediated injury.

LDLr is of primary importance in the binding and internalization of plasma-derived LDL-cholesterol and in regulating plasma LDL concentrations. Changes in receptor activity alter the rates of LDL uptake with a corresponding increase or reduction in plasma LDL levels.

The SR DKO mice used in our experiment permitted us to test the hypothesis that lipid uptake mediated by the LDLr pathway, not involving SR-A or CD36, impacts lipid accumulation in the aortas and livers of inflammatory mice. Using SR DKO inflammatory mice, our results demonstrate that lipid accumulation occurs in aortas and livers. The data in this study showed that SR may not be necessary for lipid accumulation in inflammatory mice with a decreased serum lipid level, despite the fact that SRs account for the vast majority of modified LDL uptake in cultured macrophages. In addition,

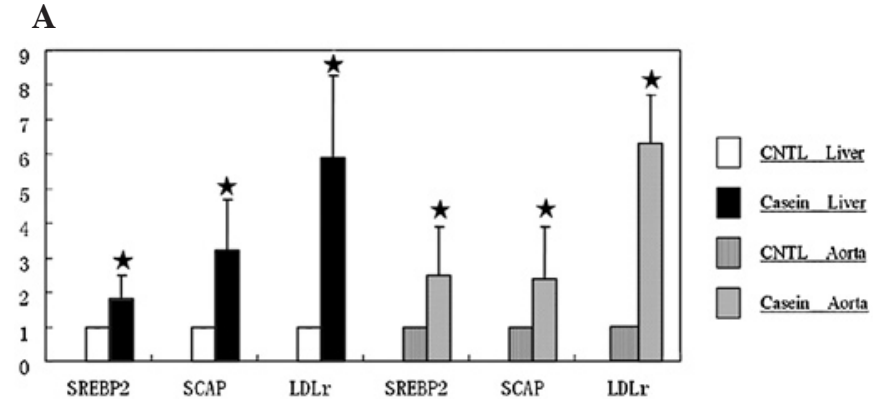

B

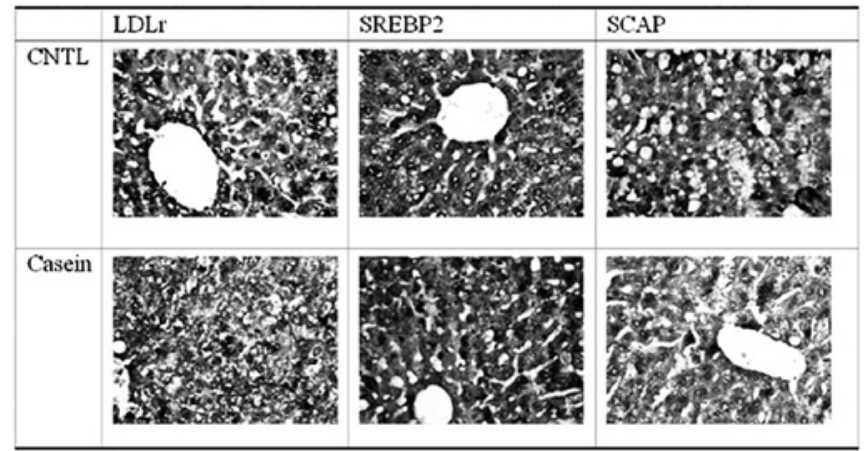

C

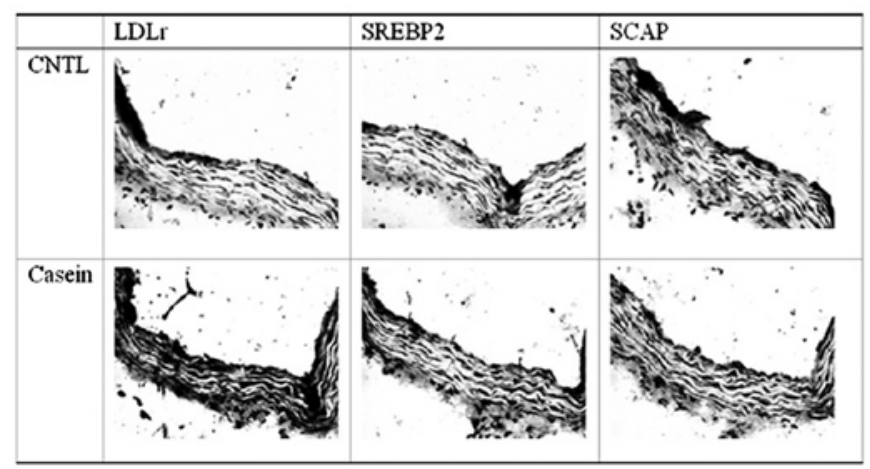

Figure 4. Effect of inflammatory stress induced by casein injection on the mRNA expression of LDLr, SREBP2 and SCAP, in the livers or aortas of SR DKO was determined by real-time PCR (A). 18SrRNA served as the house keeping gene. Results represent the mean $\pm \mathrm{SD}(\mathrm{n}=6)$. ${ }^{*} \mathrm{P}<0.01$ vs. control. The protein levels of SCAP, SREBP2 and LDLr in the livers (B) or aortas (C) of SR DKO were determined by immunohistochemical staining.

measurement of total and free cholesterol representing lipid accumulation showed increased esterified cholesterol in the livers.

Although lipid accumulation is the earliest histological finding of an atherosclerotic lesion, the mechanism by which aortas accumulate cholesterol remains unclear. Evaluation of mechanisms leading to lipid disturbances in inflammatory mice is therefore essential for a better understanding of lipidmediated injury. LDLr is positively regulated by SREBP2; we found that enhanced SREBP2 gene expression was associated with the up-regulation of SCAP and LDLr gene expression and with enhanced cholesterol uptake via the LDLr pathway in aortas and livers of SR DKO mice administered a combination of a high-fat diet and casein injection in comparison to control animals. Such dysregulation would permit SREBP2 mRNA accumulation, uncontrolled by cellular cholesterol. Increased SREBP2 in inflammatory mice may be one of the 
major causes of hypocholesterolemia and, consequently, of promoting lipid accumulation. Our results suggest that an increased SREBP2 level contributes to lipid accumulation in aortas or livers. Thus, taking into account the above information and the results presented in this study, we hypothesize that SREBP2 contributes to the development and progression of lipid accumulation in the aortas or livers of inflammatory animals.

A positive correlation between SREBP2 gene expression and the LDLr mRNA level confirms the SREBP2-dependent mechanism of LDLr transcriptional regulation. Taken together, our data displayed an important role for SREBP2 in promoting lipid accumulation and lowering serum cholesterol level via the LDLr pathway. Results of the present study suggest that lipid accumulations are associated with increase in SREBP2 gene expression in aortas or livers. Lipid dysregulation in inflammatory stress was associated with increased accumulation of lipids in aortas or livers, mediated by increased expression of SREBP2 in aortas or livers.

It is likely that up-regulation of SREBP2 gene expression in the presence of a higher cholesterol concentration in the cell is overridden in the presence of inflammatory cytokines. Thus, these data suggest that inflammatory cytokines, concentrations of which are elevated in the inflammatory state, decrease the plasma cholesterol concentration in inflammatory mice through dysregulation of SREBP2 gene expression and, consequently, through up-regulation of its target genes, including LDLr gene. Similarly, Ruan suggested that inflammatory cytokines modify cholesterol homeostasis through dysregulation of the LDLr $(12,13)$. This partly explains the association between inflammation and lipid accumulation. Moreover, it may partly explain the positive correlation between the level of SREBP2 and the abundance of LDLr receptor mRNA. Other factors which may influence the SREBP2 level in the aortas or livers in inflammatory animals cannot be excluded.

Hepatic inflammations have impacts on cholesterol uptake and may exacerbate lipid-mediated cholesterol influx to promote lipid accumulation in vascular smooth muscle or hepatic cells. First, they act in the liver, where increased SREBP2 gene expression leads to hypocholesterolemia and, secondly, to lipid accumulation in the aortas. Lipid accumulation caused by increased SREBP2 level directly contributes to the development of foam cell formation and progression of atherosclerosis. Coordinated up-regulation of SREBP2 and SCAP gene expression, with subsequently enhanced LDLr gene expression, may play a key role in lipid accumulation in aortas and livers of inflammatory mice.

In the present study, the mice were treated with casein injection for 14 weeks to induce a predictable low-grade, systemic inflammation and to test whether this inflammatory stress enhances lipid accumulation in aortas or livers. SAA in mice, like C-reactive protein in humans, is an acute-phase protein synthesized by the liver and a good inflammatory marker for systemic inflammatory stress (7). Our results showed that inflammation induced by a casein injection significantly increased serum levels of SAA, suggesting that systemic and local inflammatory stress was successfully induced in the mice. A recent study revealed that macrophages from mice lacking CD36 had decreased pro-inflammation characteristics under a pro-inflammation stimulus. Plasma levels of cytokines/chemokines showed that $\mathrm{CD}^{-/-} \mathrm{ApoE}^{-/-}$ and $\mathrm{CD}^{-/-} \mathrm{SRA}^{-/} \mathrm{ApoE}^{-/-}$mice had a less pro-inflammation phenotype compared to $\mathrm{ApoE}^{-/-}$and $\mathrm{SRA}^{-/} \mathrm{ApoE}^{-/-}$mice (14). Our data demonstrated that the SR DKO mice fed a high-fat diet exhibited a normal lipid level of lipid, while the inflammatory mice exhibited a significantly decreased lipid level, as the base of pathogenesis of lipid accumulation in aortas or livers. Histochemical examination demonstrated massive lipid accumulation in the aortic section of the inflammatory mice using Oil Red O and H\&E staining. Quantitative assay confirmed a significant lipid accumulation in the livers. These data suggest a strong association between enhanced lipid accumulation in the aortas or livers and inflammation induced by casein injection. These results strongly support that inflammation may be a critical event in the progression of lipid accumulation and may cause or further exacerbate lipid-mediated injuries in the aortas or livers of SR DKO mice.

Our previous studies demonstrated that inflammation disrupts LDLr-mediated negative feedback regulation to accelerate foam cell formation in vascular smooth muscle and mesangial cells $(12,13)$. We demonstrated that inflammatory stress increased the cholesterol accumulation via LDLr in the aortas or livers in the SR DKO mice fed a high-fat diet by increasing SREBP2 expression. We then studied the LDLr pathway to explore possible mechanisms of increased lipid accumulation under inflammatory stress. These results revealed that inflammation up-regulated the mRNA and protein expression of LDLr, SCAP and SREBP2 in the aortas or livers of the inflammatory mice compared to the controls, consistent with our previous in vivo findings in wild-type C57BL/6 mice. In addition, low-grade inflammation stress induced by casein injection may be useful for studying the effect of cholesterol metabolism on lipid accumulation. Although the levels of IL- 6 were the same between the groups with the presence or absence of inflammation since SR deficiency had a marked suppressive effect on inflammation gene expression, the levels of SAA in the serum of the inflammatory mice were significantly higher than those of the control animals. These data are consistent with previous studies on SRs to downstream inflammation signaling (15-19).

Studies have revealed that SR deficiency causes substantial changes in inflammation gene expression. Decreased expression of cytokines indicate that the loss of SRs is associated with a reduction in the inflammation milieu. In agreement with this, Kuchibhotla et al (6) reported decreased serum levels of inflammation markers in $\mathrm{ApoE}^{-/-} \mathrm{CD} 36^{-/-} \mathrm{Msrl}^{-/-}$mice, several of which overlap with our measurements of inflammation gene expression in the aortas.

Thus, a markedly increased lipid accumulation was observed in the inflammatory mice. Gene expression involving cholesterol influx increased, supporting a role for this process in triggering dysregulation of cholesterol metabolism and the development of foam cell formation in aortas.

In summary, the data in this study demonstrate that an inflammatory-induced casein injection may play a critical role in the evolution of lipid accumulation in SR DKO mice. This role appears to be mediated through their influence on the dysregulation of lipid metabolism. To fully understand the effects of loss of SR on lipid accumulation in aortas and livers, it is necessary to undertake a detailed analysis of innate 
immune systems, apoptosis and the evolution of foam cell formation in future studies.

\section{Acknowledgements}

The authors thank Ruan Xiong Zhong and Chen Ya Xi for the scientific discussion, and Zhao Lei for the excellent technical assistance. This study was supported by a grant from the National Natural Science Foundation of China (no. 30670869).

\section{References}

1. Tabas I, Williams KJ and Boren J: Subendothelial lipoprotein retention as the initiating process in atherosclerosis: update and therapeutic implications. Circulation 116: 1832-1844, 2007.

2. Kunjathoor VV, Febbraio M, Podrez EA, Moore KJ, Andersson L, Koehn S, Rhee JS, Silverstein R, Hoff HF and Freeman MW: Scavenger receptors class A-I/II and CD36 are the principal receptors responsible for the uptake of modified low density lipoprotein leading to lipid loading in macrophages. J Biol Chem 277: 49982-49988, 2002.

3. Moore KJ, Kunjathoor VV, Koehn SL, Manning JJ, Tseng AA, Silver JM, McKee M and Freeman MW: Loss of receptor-mediated lipid uptake via scavenger receptor A or CD36 pathways does not ameliorate atherosclerosis in hyperlipidemic mice. J Clin Invest 115: 2192-2201, 2005.

4. Kruth HS, Jones NL, Huang W, Zhao B, Ishii I, Chang J, Combs CA, Malide D and Zhang WY: Macropinocytosis is the endocytic pathway that mediates macrophage foam cell formation with native low density lipoprotein. J Biol Chem 280 2352-2360, 2005.

5. Wooton-Kee CR, Boyanovsky BB, Nasser MS, de Villiers WJ and Webb NR: Group V sPLA2 hydrolysis of low-density lipoprotein results in spontaneous particle aggregation and promotes macrophage foam cell formation. Arterioscler Thromb Vasc Biol 24: 762-767, 2004.

6. Kuchibhotla S, Vanegas D, Kennedy DJ, Guy E, Nimako G, Morton RE and Febbraio M: Absence of CD36 protects against atherosclerosis in ApoE knock-out mice with no additional protection provided by absence of scavenger receptor A Cardiovasc Res 78: 185-196, 2008.

7. Ma KL, Ruan XZ, Powis SH, Chen YX, Moorhead JF and Varghese Z: Inflammation stress exacerbates lipid accumulation in hepatic cells and fatty livers of apolipoprotein E knockout mice. Hepatology 48: 770-781, 2008.
8. Hillgartner FB, Salati LM and Goodridge AG: Physiological and molecular mechanisms involved in nutritional regulation of fatty acid synthesis. Physiol Rev 1: 47-76, 1995

9. Nohturfft A, DeBose-Boyd RA, Scheek S, Goldstein JL and Brown MS: Sterols regulate cycling of SREBP cleavageactivating protein (SCAP) between endoplasmic reticulum and Golgi. Proc Natl Acad Sci USA 20: 11235-11240, 1999.

10. Horton JD, Goldstein JL and Brown MS: SREBPs: activators of the complete program of cholesterol and fatty acid synthesis in the liver. J Clin Invest 109: 1125-1131, 2002.

11. Gamble W, Vaughan M, Kruth HS and Avigan J: Procedure for determination of free and total cholesterol in micro- or nanogram amounts suitable for studies with cultured cells. J Lipid Res 8: 1068-1070, 1978.

12. Ruan XZ, Varghese Z, Powis SH and Moorhead JF: Dysregulation of LDL receptor under the influence of inflammation cytokines: a new pathway for foam cell formation. Kidney Int 5: 1716-1725, 2001.

13. Ruan XZ, Moorhead JF, Tao JL, Ma KL, Wheeler DC and Powis SH: Mechanisms of dysregulation of low-density lipoprotein receptor expression in vascular smooth muscle cells by inflammation cytokines. Arterioscler Thromb Vasc Biol 5: $1150-1155,2006$

14. Manning-Tobin JJ, Moore KJ, Seimon TA, Bell SA, Sharuk M, Alvarez-Leite JI, Winther MP, Tabas I and Freeman MW: Loss of SR-A and CD36 activity reduces atherosclerotic lesion complexity without abrogating foam cell formation in hyperlipidemic mice. Arterioscler Thromb Vasc Biol 29: 19-26, 2009.

15. Devries ST, Li Y, Yao PM, Stone E, Wang Y, Davis RJ, Flavell R and Tabas I: Cholesterol induced macrophage apoptosis requires ER stress pathways and engagement of the type A scavenger receptor. J Cell Biol 171: 61-73, 2005.

16. Seimon TA, Obstfeld A, Moore KJ, Golenbock DT and Tabas I: Combinatorial pattern recognition receptor signaling alters the balance of life and death in macrophages. Proc Natl Acad Sci USA 103: 19794-19799, 2006.

17. Moore KJ, El Khoury J, Medeiros LA, Terada K, Geula C, Luster AD and Freeman MW: A CD36-initiated signaling cascade mediates inflammation effects of beta-amyloid. J Biol Chem 277: 47373-47379, 2002

18. Stuart LM, Bell SA, Stewart CR, Silver JM, Richard J, Goss JL, Tseng AA, Zhang A, El Khoury JB and Moore KJ: CD36 signals to the actin cytoskeleton and regulates microglial migration via a p130C as complex. J Biol Chem 282: 27392-27401, 2007.

19. Stuart LM, Deng J, Silver JM, Takahashi K, Tseng AA, Hennessy EJ, Ezekowitz RA and Moore KJ: Response to Staphylococcus aureus requires CD36-mediated phagocytosis triggered by the $\mathrm{COOH}$ terminal cytoplasmic domain. J Cell Biol 170: 477-485, 2005. 